\title{
TOPOLOGICAL STRUCTURE OF SOLUTION SET FOR A CLASS OF FRACTIONAL NEUTRAL EVOLUTION EQUATIONS ON THE HALF-LINE
}

\author{
Le Hoan HoA - Nguyen Ngoc Trong - Le Xuan Truong
}

\begin{abstract}
A topological structure of the set of all mild solutions of fractional neutral evolution equations with finite delay on the half-line is investigated. We show that the solution set is an $R_{\delta}$-set. It is proved on compact intervals by establishing a result on topological structure of fixed point set of Krasnosel'skiu type operators. Next, using the inverse limit method, we obtain the same result on the half-line.
\end{abstract}

\section{Introduction}

Throughout this paper $E$ denotes a Banach space endowed with the norm $|\cdot|$. Suppose that $A: D(A) \subset E \rightarrow E$ is the infinitesimal generator of an analytic semigroup $\{T(t)\}_{t \geq 0}$ of operators on $E$ and $\psi:[-r, 0] \rightarrow E$ is a function belonging to the phase space $\mathcal{C}_{0}=C([-r, 0] ; E)$. The main purpose of this paper is to study the topological structure of the set of all mild solutions of fractional neutral evolution equations with finite delay of the form

$$
\begin{cases}{ }^{\mathrm{C}} D^{q}\left[x(t)-h\left(t, x(t), x_{t}\right)\right]=A x(t)+f\left(t, x(t), x_{t}\right), & t>0, \\ x_{0}(t)=\psi(t), & t \in[-r, 0],\end{cases}
$$

2010 Mathematics Subject Classification. 34A12, 34G20, 34A08.

Key words and phrases. Topology structure; solution set; fractional differential equation; Krasnosel'skiǔ type operator. 
where ${ }^{\mathrm{C}} D^{q}$ is the Caputo fractional derivative of order $q \in(0,1)$, the histories $x_{t}:[-r, 0] \rightarrow E$ are defined by $x_{t}(s)=x(t+s)$ for all $s \in[-r, 0]$, and the functions $h, f: \mathbb{R}_{+} \times E \times \mathcal{C}_{0} \rightarrow E$ are given functions satisfying some conditions specified later.

Problem (1.1) on a compact interval has been studied by Zhou and Jiao [28] under a more general framework, namely the condition $x_{0}=\psi$ was replaced by the condition

$$
x_{0}(t)+g\left(x_{t_{1}}, \ldots, x_{t_{n}}\right)(t)=\psi(t), \quad t \in[-r, 0] .
$$

They proved an existence result by using the Krasnosel'skil fixed point theorem. In this paper we establish the existence results for problem (1.1) on the half-line and, in particular, we prove that the mild solution set of this problem is an $\mathrm{R}_{\delta}$-set. For the reader's convenience, we recall here some definitions.

Definition 1.1 (see [14]). Let $M$ be a topological space. Then

(a) $M$ is called an absolute retract if each continuous map $f: B \rightarrow M$, where $B$ is a closed subset of some topological space $N$, possesses a continuous extension over $N$.

(b) $M$ is called an $R_{\delta}$-set if it is homeomorphic to the intersection of a decreasing sequence of compact absolute retracts.

Note that any $\mathrm{R}_{\delta}$-set is a nonempty compact connected space. On the other hand, it is acyclic with respect to the Čech homology functor, i.e. it has the same homology as the one point space. It may be not a singleton but, from the point of view of algebraic topology, it is equivalent to a point (see [14]).

In 1890, Peano proved that the Cauchy problem

$$
\left\{\begin{array}{l}
x^{\prime}(t)=f(t, x(t)), \quad 0<t \leq a, \\
x(0)=x_{0}
\end{array}\right.
$$

where $f:[0, a] \times \mathbb{R}^{n} \rightarrow \mathbb{R}^{n}$ is continuous, has local solutions although the uniqueness property does not hold in general. This observation became a motivation for studying the structure of the solution set, Sol, for problem (1.2). Peano also proved that, in the case $n=1$, the set $\operatorname{Sol}(t)=\{x(t): x \in \operatorname{Sol}\}$ is nonempty, compact and connected in the standard topology of the real line, for $t$ in some neighbourhood of 0 . In 1923, Kneser generalized this result for arbitrary $n$. Next, in 1928, Hukuhara proved that Sol is a continuum in the Banach space of continuous functions with sup norm. A more precise characterization of Sol has been found in 1942 by Aronszajn [2]. He proved that Sol is an $\mathrm{R}_{\delta}$-set. So Sol is acyclic. The analogous result was obtained for upper-Carathéodory inclusions by De Blasi and Myjak in [8]. For more details, historical remarks and related references, see [1]. 
In many cases, solution sets for differential problems correspond with fixed point sets of operators in suitable function spaces. The results on the $\mathrm{R}_{\delta}$ property of fixed point sets often require compactness of operators on the whole linear space. This is a difficulty in application. In this paper we first establish a result on the $\mathrm{R}_{\delta}$ property of the fixed point set for Krasnosel'skil type operators of the form $\mathcal{U}+\mathcal{B}$, where $\mathcal{U}$ is a contraction operator and $\mathcal{B}$ is a completely continuous operator (see Theorem 3.2). Then, by using this result and the inverse limit method, we not only obtain the existence result but also the $\mathrm{R}_{\delta}$ property for the mild solution set of problem (1.1).

For more results on fractional differential equation we refer the interested reader to [4], [5], [9]-[12], [18], [23], [24], [26], [28] and on topological structure of solution sets we refer to [3], [6], [7], [13]-[15], [20]-[22], [27].

\section{Preliminaries and assumptions}

For fractional calculus we recall that if $x:[0,+\infty) \subset \mathbb{R} \rightarrow E$ then

- the fractional integral of order $\alpha>0$ with the lower limit zero for $x$ is defined as

$$
I^{\alpha} x(t):=\frac{1}{\Gamma(\alpha)} \int_{0}^{t}(t-s)^{\alpha-1} x(s) d s, \quad t>0,
$$

and

- the Caputo fractional derivative of order $\alpha>0$ for the function $x$ is defined by

$$
{ }^{\mathrm{C}} D^{\alpha} x(t):=I^{n-\alpha} x^{(n)}(t)=\frac{1}{\Gamma(n-\alpha)} \int_{0}^{t}(t-s)^{n-\alpha-1} x^{(n)}(s) d s,
$$

provided that the right-hand sides of above equalities are pointwise defined on $[0, \infty)$. We also note that the integrals which appear in these definitions are taken in Bochner's sense.

For the operator $A$, we assume that it is the infinitesimal generator of an analytic semigroup $\{T(t)\}_{t \geq 0}$ such that $0 \in \rho(A)$, where $\rho(A)$ is the resolvent set of $A$. It is well known that, for each $\eta \in(0,1]$, the fractional power $A^{\eta}$ is defined as a closed linear operator on its domain $D\left(A^{\eta}\right)$. The following properties will be used (see [25]):

(i) There is a constant $M \geq 1$ such that $M:=\sup _{t \in \mathbb{R}_{+}}|T(t)|<\infty$.

(ii) $D\left(A^{\eta}\right)$ is a Banach space with the norm $\|x\|_{\eta}=\left|A^{\eta} x\right|$, for $x \in D\left(A^{\eta}\right)$.

(iii) $T(t): E \rightarrow D\left(A^{\eta}\right)$, for all $t \geq 0$.

(iv) $A^{\eta} T(t) x=T(t) A^{\eta} x$, for each $x \in D\left(A^{\eta}\right)$ and $t \geq 0$.

(v) For every $t>0, A^{\eta} T(t)$ is bounded on $E$ and there exists $C_{\eta}>0$ such that $\left|A^{\eta} T(t)\right| \leq C_{\eta} / t^{\eta}$.

(vi) $A^{-\eta}$ is a bounded linear operator for $0 \leq \eta \leq 1$ in $E$. 
Definition 2.1 ([28]). By a mild solution of problem (1.1) we mean a continuous function $x:[-r, \infty) \rightarrow E$ which satisfies $x_{0}=\psi$ on $[-r, 0]$ and

$$
\begin{aligned}
x(t)= & S_{q}(t)\left[\psi(0)-h\left(0, \psi(0), x_{0}\right)\right]+h\left(t, x(t), x_{t}\right) \\
& +\int_{0}^{t}(t-s)^{q-1} A T_{q}(t-s) h\left(s, x(s), x_{s}\right) d s \\
& +\int_{0}^{t}(t-s)^{q-1} T_{q}(t-s) f\left(s, x(s), x_{s}\right) d s, \quad t \in \mathbb{R}_{+},
\end{aligned}
$$

where the operators $\left\{S_{q}(t)\right\}_{t \geq 0}$ and $\left\{T_{q}(t)\right\}_{t \geq 0}$ are given by

$$
S_{q}(t) x=\int_{0}^{\infty} \phi_{q}(\theta) T\left(t^{q} \theta\right) x d \theta \quad \text { and } \quad T_{q}(t) x=q \int_{0}^{\infty} \theta \phi_{q}(\theta) T\left(t^{q} \theta\right) x d \theta,
$$

with $\phi_{q}$ a probability density function defined on $(0, \infty)$ by

$$
\begin{gathered}
\phi_{q}(\theta)=\frac{1}{q} \theta^{-1-1 / q} \psi_{q}\left(\theta^{-1 / q}\right) \geq 0 \\
\psi_{q}(\theta)=\frac{1}{\pi} \sum_{n=1}^{\infty}(-1)^{n-1} \theta^{-n q-1} \frac{\Gamma(n q+1)}{n !} \sin (n \pi q), \quad \theta \in(0, \infty) .
\end{gathered}
$$

The following results on $S_{q}$ and $T_{q}$ are used throughout this paper.

Lemma $2.2([28])$. The operators $S_{q}$ and $T_{q}$ have the following properties:

(a) For any fixed $t \geq 0, S_{q}(t)$ and $T_{q}(t)$ are linear and bounded operators. Further, for all $x \in E$, we have

$$
\left|S_{q}(t) x\right| \leq M|x| \quad \text { and } \quad\left|T_{q}(t) x\right| \leq \frac{q M}{\Gamma(1+q)}|x| .
$$

(b) Operators $\left\{S_{q}(t)\right\}_{t \geq 0}$ and $\left\{T_{q}(t)\right\}_{t \geq 0}$ are strongly continuous, i.e. for all $x \in E$ and $0 \leq t_{1}<t_{2}$ we have

$$
\left|S_{q}\left(t_{1}\right) x-S_{q}\left(t_{2}\right) x\right| \rightarrow 0 \quad \text { and } \quad\left|T_{q}\left(t_{1}\right) x-T_{q}\left(t_{2}\right) x\right| \rightarrow 0 \quad \text { as } t_{1} \rightarrow t_{2} .
$$

(c) If $T(t)$ is a compact operator for every $t>0$ then $S_{q}(t)$ and $T_{q}(t)$ are also compact operators for every $t>0$.

(d) For any $x \in E, \alpha \in(0,1), \beta \in(0,1]$, we have

$$
\begin{array}{cc}
A T_{q}(t) x=A^{1-\beta} T_{q}(t) A^{\beta} x, & t \in \mathbb{R}_{+}, \\
\left|A^{\alpha} T_{q}(t)\right| \leq \frac{q C_{\alpha} \Gamma(2-\alpha)}{\Gamma(1+q(1-\alpha))} t^{-\alpha q}, & t>0 .
\end{array}
$$

Now assume that $F$ is a Fréchet space whose topology is given by the family of seminorms $\left\{p_{n}: n \in \mathbb{N}\right\}$.

Definition 2.3. Let $\left\{k_{n}\right\}_{n=1}^{\infty}$ be a sequence in $[0,1)$. The map $\mathcal{U}: F \rightarrow F$ is said to be a $k_{n}$-contraction operator if

$$
p_{n}(\mathcal{U}(x)-\mathcal{U}(y)) \leq k_{n} p_{n}(x-y), \quad \text { for all } x, y \in F
$$


The following fact will be used to prove our main results.

Lemma 2.4 ([16], [17]). Let two operators $\mathcal{U}, \mathcal{B}: F \rightarrow F$ be given. Assume that the following conditions hold:

(a) $\mathcal{U}$ is a $k_{n}$-contraction operator, for every $n \in \mathbb{N}$,

(b) $\mathcal{B}$ is a completely continuous operator and

$$
\lim _{p_{n}(x) \rightarrow \infty} \frac{p_{n}(\mathcal{B}(x))}{p_{n}(x)}=0, \quad \text { for all } n \in \mathbb{N} .
$$

Then $\mathcal{U}+\mathcal{B}$ has a fixed point in $F$.

\section{Topological structure of fixed point set for Krasnosel'skiǐ type operators}

The following lemma is a corollary of [13, Theorem 2.1].

Lemma 3.1. Let $(X,\|\cdot\|)$ be a Banach space and $K: X \rightarrow X$ be a completely continuous map. Assume that there is a sequence of completely continuous maps $K_{m}: X \rightarrow X$ such that

(a) $\left\|K_{m}(x)-K(x)\right\|<1 / m$, for all $x \in X$ and for all $m \in \mathbb{N}$,

(b) for each $m \geq 1$ and each $y \in X$ with $\|y\|<1 / m$, the equation $x=$ $K_{m}(x)+y$ has exactly one solution.

Then $\operatorname{Fix}(K)$, the set of all fixed points of $K$, is an $R_{\delta}$-set.

Next we shall prove an important result of this paper.

Theorem 3.2. Let $(X,\|\cdot\|)$ be a Banach space and $\mathcal{U}, \mathcal{B}: X \rightarrow X$ be two operators. Assume that

(a) $\mathcal{U}$ is a $k$-contraction operator,

(b) $\mathcal{B}$ is a completely continuous operator satisfying the following conditions:

(i) $\lim _{\|x\| \rightarrow \infty}\|\mathcal{B}(x)\| /\|x\|=0$,

(ii) there is a sequence of completely continuous operators $\mathcal{B}_{n}: X \rightarrow X$ $(n=1,2, \ldots)$ such that for any bounded subset $\mathcal{D}$ of $X$ we have

$$
\lim _{n \rightarrow \infty} \sup _{x \in \mathcal{D}}\left\|\mathcal{B}_{n}(x)-\mathcal{B}(x)\right\|=0,
$$

(c) for every $h \in X$ with $\|h\|<1 / n$ the equation $x=\mathcal{U}(x-h)+\mathcal{B}_{n}(x)+h$ has at most one solution.

Then $\operatorname{Fix}(\mathcal{U}+\mathcal{B})=\{x \in X: x=\mathcal{U} x+\mathcal{B} x\}$ is an $R_{\delta}$-set.

PROOF. It follows from assumption (a) that the operator $I-\mathcal{U}$ is invertible and its inverse is uniformly continuous on $X$. We define the completely continuous operator $f: X \rightarrow X$ by

$$
f(x)=(I-\mathcal{U})^{-1} \mathcal{B}(x), \quad \text { for } x \in X .
$$


Our proof consists of three steps.

Step 1. There exists a convex closed and bounded subset $\mathcal{D}$ of $X$ such that $f(\mathcal{D}) \subset \mathcal{D}$.

For each $z \in X$ we define the operator $\mathcal{U}_{z}: X \rightarrow X$ by

$$
\mathcal{U}_{z}(x)=\mathcal{U}(x)+z,
$$

and $\mathcal{U}_{z}^{m}=\left(\mathcal{U}_{z}\right)^{m}$, for $m \in \mathbb{N}$. Then, from [16, Theorem 10], it follows that $\mathcal{U}_{z}$ admits a unique fixed point being $(I-\mathcal{U})^{-1}(z)$ and we have:

- the sequence $\left\{\mathcal{U}_{z}^{m}(x)\right\}_{m=1}^{\infty}$ converges to $(I-\mathcal{U})^{-1}(z)$ for all $x \in X$, that is,

$$
\lim _{m \rightarrow \infty}\left\|\mathcal{U}_{z}^{m}(x)-(I-\mathcal{U})^{-1}(z)\right\|=0,
$$

- there exists $\alpha>1$ such that

$$
\left\|\mathcal{U}_{z}^{m}\left(x_{0}\right)-x_{0}\right\| \leq \alpha\|z\|, \quad \text { for all } m \in \mathbb{N},
$$

where $x_{0}$ is the unique fixed point of $\mathcal{U}$.

Next, it follows from assumption (b) that there exists $\rho^{1}>0$ satisfying

$$
\|\mathcal{B}(x)\|<\frac{1}{2 \alpha}\left\|x-x_{0}\right\|
$$

provided that $\left\|x-x_{0}\right\|>\rho^{1}$. Then we choose a positive constant $\rho$ such that $\rho>\rho^{1}+\alpha(1+\beta)+2$, where $\beta=\sup \left\{\|\mathcal{B} x\|: x \in X\right.$ with $\left.\left\|x-x_{0}\right\| \leq \rho^{1}\right\}$. It is necessary to note that $\beta \neq \infty$ thanks to complete continuity of the operator $\mathcal{B}$.

Now we set $\mathcal{D}=\left\{x \in X:\left\|x-x_{0}\right\| \leq \rho\right\}$. Then $\mathcal{D}$ is a convex closed bounded subset of $X$. Moreover, it is not difficult to prove that $\mathcal{U}_{\mathcal{B}(x)}^{m}\left(x_{0}\right) \in \mathcal{D}$, for all $x \in \mathcal{D}$. Consequently, passing to the limit as $m \rightarrow \infty$ and using (3.1) we have $f(\mathcal{D})=(I-\mathcal{U})^{-1} \mathcal{B}(\mathcal{D}) \subset \mathcal{D}$.

Step 2. Let $f_{\mathcal{D}}$ be the restriction of $f$ on $\mathcal{D}$. We will show that

$$
\operatorname{Fix}(\mathcal{U}+\mathcal{B})=\operatorname{Fix}\left(f_{\mathcal{D}}\right) .
$$

For this, it is sufficient to prove that if $x$ is a fixed point of $\mathcal{U}+\mathcal{B}$ then $x \in \mathcal{D}$. Indeed, if $x \in \operatorname{Fix}(\mathcal{U}+\mathcal{B})$ then $x$ is also the unique fixed point of $\mathcal{U}_{\mathcal{B}(x)}$ and

$$
\lim _{m \rightarrow \infty} \mathcal{U}_{\mathcal{B}(x)}^{m}\left(x_{0}\right)=x .
$$

By letting $m \rightarrow \infty$ in (3.2), we obtain $\left\|x-x_{0}\right\| \leq \alpha\|\mathcal{B} x\|$. If $\left\|x-x_{0}\right\|>\rho$ then $\left\|x-x_{0}\right\|<\left\|x-x_{0}\right\| / 2$. This contradiction implies that $\operatorname{Fix}(\mathcal{U}+\mathcal{B}) \subset \mathcal{D}$.

Step 3. It follows from Step 1 and Schauder's fixed point theorem that $\operatorname{Fix}\left(f_{\mathcal{D}}\right) \neq \emptyset$. Now we put $f_{n}=(I-\mathcal{U})^{-1} \mathcal{B}_{n}$. Then $f_{n}$ is clearly completely continuous. On the other hand, since $(I-\mathcal{U})^{-1}$ is uniformly continuous on $X$, 
for each $n \in \mathbb{N}$, there exists $\delta_{n} \in(0,1 / 2)$ and $\delta_{n} \rightarrow 0$ such that for all $x, y \in X$ and $\|x-y\|<\delta_{n}$ we have

$$
\left\|(I-\mathcal{U})^{-1}(x)-(I-\mathcal{U})^{-1}(y)\right\|<\frac{1}{n} .
$$

From assumption (b) (ii) there exists a subsequence of $\left\{\mathcal{B}_{n}\right\}$ which is still denoted by $\mathcal{B}_{n}$ such that

$$
\sup _{x \in D}\left\|\mathcal{B}_{n}(x)-\mathcal{B}(x)\right\|<\delta_{n} .
$$

Combining (3.5) and (3.6) we deduce $\left\|f_{n}(x)-f(x)\right\|<1 / n$, for all $x \in \mathcal{D}$.

Finally it is noted that, for $y \in X$ satisfying $\|y\|<1 / n$, the equation $x=$ $f_{n}(x)+y$ is equivalent to the equation

$$
x=\mathcal{U}(x-y)+\mathcal{B}_{n}(x)+y .
$$

In order to prove the existence of a solution for equation (3.7), by assumption (iii), we need only to check that this equation has at least one solution. For this purpose we consider the operator $\mathcal{F}: X \rightarrow X$ defined by $\mathcal{F}(x)=\mathcal{U}(x-y)+y$. Then it is easy to see that $\mathcal{F}$ is a $k$-contraction operator and $x_{0}+y$ is the unique fixed point of $\mathcal{F}$. Moreover, if $z \in X$ then we have

$$
\mathcal{F}_{z}^{m}(x)=\mathcal{U}_{z}^{m}(x-y)+y,
$$

for all $m \in \mathbb{N}^{*}$ and for all $x \in X$. Hence, it follows from (3.2) that

$$
\begin{aligned}
\| \mathcal{F}_{\mathcal{B}_{n}(x)}^{m}( & \left.x_{0}+y\right)-x_{0} \| \\
& =\left\|\mathcal{U}_{\mathcal{B}_{n}(x)}^{m}\left(x_{0}\right)+y-x_{0}\right\| \leq\left\|\mathcal{U}_{\mathcal{B}_{n}(x)}^{m}\left(x_{0}\right)-x_{0}\right\|+\|y\| \\
& \leq \alpha\left\|\mathcal{B}_{n}(x)\right\|+1 \leq \alpha\left\|\mathcal{B}_{n}(x)-\mathcal{B}(x)\right\|+\alpha\|\mathcal{B}(x)\|+1 .
\end{aligned}
$$

Combining (3.6) and (3.8), we get

$$
\left\|\mathcal{F}_{\mathcal{B}_{n}(x)}^{m}\left(x_{0}+y\right)-x_{0}\right\| \leq \frac{\alpha}{2}+\alpha\|\mathcal{B}(x)\|+1 .
$$

We consider two cases:

Case 1. $\left\|x-x_{0}\right\| \leq \rho^{1}$. In this case we have

$$
\left\|\mathcal{F}_{\mathcal{B}_{n}(x)}^{m}\left(x_{0}+y\right)-x_{0}\right\| \leq \frac{\alpha}{2}+\alpha \beta+1<\rho .
$$

Case 2. $\rho^{1}<\left\|x-x_{0}\right\| \leq \rho$. Then we have $\rho / 2>\alpha / 2+1$. Hence

$$
\left\|\mathcal{F}_{\mathcal{B}_{n}(x)}^{m}\left(x_{0}+y\right)-x_{0}\right\| \leq \frac{\alpha}{2}+\frac{\left\|x-x_{0}\right\|}{2}+1<\rho .
$$

This implies that $(I-\mathcal{F})^{-1} \mathcal{B}_{n}(\mathcal{D}) \subset \mathcal{D}$. By using again Schauder's fixed point theorem, $\mathcal{F}+\mathcal{B}_{n}$ has a fixed point $x \in D$ which is a solution of equation (3.7). Therefore, by applying Lemma 3.2, the proof of the theorem is complete. 
Corollary 3.3. Let $X=C([a, b] ; E)$ be the Banach space of all continuous functions on $[a, b]$ taking values in a Banach space $(E ;|\cdot|)$ and endowed with the sup norm $\|\cdot\|$. Let $\mathcal{U}: X \rightarrow X$ be a $k$-contraction operator, $\mathcal{B}: X \rightarrow X$ be a completely continuous operator such that

$$
\lim _{\|x\| \rightarrow \infty} \frac{\|\mathcal{B}(x)\|}{\|x\|}=0 .
$$

Assume that there exist $t_{0} \in[a, b]$ and $e_{0} \in E$ satisfying the following conditions:

(a) $\mathcal{B}(x)\left(t_{0}\right)=e_{0}$ for all $x \in X$,

(b) for any $\varepsilon>0$, if $\left.x\right|_{I_{\varepsilon}}=\left.y\right|_{I_{\varepsilon}}$, then $\left.\mathcal{U}(x)\right|_{I_{\varepsilon}}=\left.\mathcal{U}(y)\right|_{I_{\varepsilon}}$ and $\left.\mathcal{B}(x)\right|_{I_{\varepsilon}}=$ $\left.\mathcal{B}(y)\right|_{I_{\varepsilon}}$, where $I_{\varepsilon}=[a, b] \cap\left[t_{0}-\varepsilon, t_{0}+\varepsilon\right]$.

Then $\operatorname{Fix}(\mathcal{U}+\mathcal{B})$ is an $R_{\delta}$-set.

Proof. For each $n \in \mathbb{N}$, we define $b_{n}:[a, b] \rightarrow[a, b]$ by

$$
b_{n}(t)= \begin{cases}t_{0} & \text { if }\left|t-t_{0}\right| \leq \frac{1}{n} \\ t-\frac{1}{n\left|t-t_{0}\right|}\left(t-t_{0}\right) & \text { if }\left|t-t_{0}\right| \geq \frac{1}{n}\end{cases}
$$

and consider the operator $\mathcal{B}_{n}: X \rightarrow X$ defined by

$$
\mathcal{B}_{n}(x)(t)=\mathcal{B}(x)\left(b_{n}(t)\right), \quad t \in[a, b] .
$$

First, we will prove that $\mathcal{B}_{n}$ is completely continuous and for each bounded set $\mathcal{D}$ in $X$ we have

$$
\lim _{n \rightarrow \infty} \sup _{x \in \mathcal{D}}\left\|\mathcal{B}_{n}(x)-\mathcal{B}(x)\right\|=0 .
$$

It is not difficult to see that $\mathcal{B}_{n}$ is continuous.

Now, let $\mathcal{D}$ be a bounded subset of $X$. Since $\mathcal{B}$ is completely continuous the set $\mathcal{B}(\mathcal{D})$ is relatively compact. Hence, for every $t \in[a, b]$,

$$
\mathcal{B}_{n}(\mathcal{D})(t):=\left\{\mathcal{B}_{n}(x)(t)=\mathcal{B}(x)\left(b_{n}(t)\right): x \in \mathcal{D}\right\}
$$

is also relatively compact. On the other hand, thanks to the equicontinuity of $\mathcal{B}(\mathcal{D})$ we deduce that for any $\varepsilon>0$, there exists $\delta>0$ such that

$$
\left|\mathcal{B}(x)\left(t_{1}\right)-\mathcal{B}(x)\left(t_{2}\right)\right|<\varepsilon,
$$

for all $x \in \mathcal{D}$ and for all $t_{1}, t_{2} \in[a, b]$ satisfying $\left|t_{1}-t_{2}\right|<\delta$. By the uniform continuity of $b_{n}$ on $[a, b]$, we can choose $\delta^{\prime}>0$ such that $\left|b_{n}\left(t_{1}\right)-b_{n}\left(t_{2}\right)\right|<\delta$ if $\left|t_{1}-t_{2}\right|<\delta^{\prime}$. This implies that $\mathcal{B}_{n}(\mathcal{D})$ is equicontinuous on $[a, b]$. Therefore, $\mathcal{B}_{n}(\mathcal{D})$ is relatively compact. And so $\mathcal{B}_{n}$ is completely continuous on $X$. Now we choose $n_{0} \in \mathbb{N}$ such that $1 / n_{0}<\delta$. Since $\left|b_{n}(t)-t\right|<1 / n$, for all $t \in[a, b]$ we deduce that

$$
\left|\mathcal{B}_{n}(x)(t)-\mathcal{B}(x)(t)\right|=\left|\mathcal{B}(x)\left(b_{n}(t)\right)-\mathcal{B}(x)(t)\right|<\varepsilon
$$


for all $x \in \mathcal{D}, t \in[a, b]$ and for all $n \geq n_{0}$. This implies

$$
\sup _{x \in \mathcal{D}}\left\|\mathcal{B}_{n}(x)-\mathcal{B}(x)\right\| \leq \varepsilon, \quad \text { for all } n \geq n_{0} .
$$

Next, it is necessary to note that, for $y \in X$ such that $\|y\|<1 / n$, the perturbed equation $x=\mathcal{U}(x-y)+\mathcal{B}_{n}(x)+y$ is equivalent to the equation $f_{n}(x)=y$, where

$$
f_{n}=I-(I-\mathcal{U})^{-1} \mathcal{B}_{n}, \quad n \in \mathbb{N} .
$$

Let $x \in X$ and $t \in I_{1 / n}$ be arbitrary. It follows from assumption (a) that

$$
\mathcal{B}_{n}(x)(t)=\mathcal{B}(x)\left(b_{n}(t)\right)=\mathcal{B}(x)\left(t_{0}\right)=e_{0} .
$$

And by using (b), this implies

$$
\mathcal{U}_{\mathcal{B}_{n}(x)}(0)(t)=\mathcal{U}(0)(t)+\mathcal{B}_{n}(x)(t)=\mathcal{U}(0)(t)+e_{0}=: e(t) .
$$

Then we have

$$
\mathcal{U}_{\mathcal{B}_{n}(x)}^{2}(0)(t)=\mathcal{U}\left(\mathcal{U}_{\mathcal{B}_{n}(x)}(0)\right)(t)+\mathcal{B}_{n}(x)(t)=\mathcal{U}(e)(t)+e_{0}=\mathcal{U}_{e_{0}}(e)(t),
$$

where $e_{0}(t):=e_{0}$ for all $t \in[a, b]$. Similarly, it is easy to obtain the equality

$$
\mathcal{U}_{\mathcal{B}_{n}(x)}^{m+1}(0)(t)=\mathcal{U}_{e_{0}}^{m}(e)(t)
$$

So, by passing to the limit as $m \rightarrow \infty$,

$$
(I-\mathcal{U})^{-1} \mathcal{B}_{n}(x)(t)=(I-\mathcal{U})^{-1}\left(e_{0}\right)(t),
$$

for all $x \in X$ and $t \in I_{1 / n}$. Now let $k \in\{2,3, \ldots\}$ and let $x, z \in X$ satisfy the condition $x(t)=z(t)$, for all $t \in I_{(k-1) / n}$. We shall prove that, for all $m \in \mathbb{N}$,

$$
\left.\mathcal{U}_{\mathcal{B}_{n}(x)}^{m}\left(e_{0}\right)\right|_{I_{k / n}}=\left.\mathcal{U}_{\mathcal{B}_{n}(z)}^{m}\left(e_{0}\right)\right|_{I_{k / n}} .
$$

Indeed, we first note that if $t \in I_{k / n}$ then $b_{n}(t) \in I_{(k-1) / n}$ by $\left|b_{n}(t)-t_{0}\right|=$ $\left|t-t_{0}\right|-1 / n$. Hence,

$$
\mathcal{B}_{n}(x)(t)=\mathcal{B}(x)\left(b_{n}(t)\right)=\mathcal{B}(z)\left(b_{n}(t)\right)=\mathcal{B}_{n}(z)(t),
$$

for all $t \in I_{k / n}$. This implies that

$$
\mathcal{U}_{\mathcal{B}_{n}(x)}\left(e_{0}\right)(t)=\mathcal{U}_{\mathcal{B}_{n}(z)}\left(e_{0}\right)(t), \quad \text { for all } t \in I_{k / n} .
$$

A simple inductive argument implies that

$$
\mathcal{U}_{\mathcal{B}_{n}(x)}^{m}\left(e_{0}\right)(t)=\mathcal{U}_{\mathcal{B}_{n}(z)}^{m}\left(e_{0}\right)(t), \quad \text { for all } t \in I_{k / n} .
$$

By passing to the limit as $m \rightarrow \infty$, we obtain

$$
(I-\mathcal{U})^{-1} \mathcal{B}_{n}(x)(t)=(I-\mathcal{U})^{-1} \mathcal{B}_{n}(z)(t),
$$

for all $t \in I_{k / n}$. So we have proved that

(3.11) $\left.x\right|_{I_{(k-1) / n}}=\left.\left.z\right|_{I_{(k-1) / n}} \Rightarrow\left((I-\mathcal{U})^{-1} \mathcal{B}_{n}(x)\right)\right|_{I_{k / n}}=\left.\left((I-\mathcal{U})^{-1} \mathcal{B}_{n}(z)\right)\right|_{I_{k / n}}$. 
Finally, it is sufficient to prove that $f_{n}$ is injective for all $n \in \mathbb{N}$. In fact, assume that for some $x, z \in X$ we have $f_{n}(x)=f_{n}(z)$. This implies that

$$
x-z=(I-\mathcal{U})^{-1} \mathcal{B}_{n}(x)-(I-\mathcal{U})^{-1} \mathcal{B}_{n}(z) .
$$

By combining (3.10) and (3.11), we deduce that $x=z$.

\section{Structure of the solution set on the half-line}

4.1. Notations. In this section, for $\sigma \geq 0$, we use the notations

$$
\mathbb{N}_{\sigma}:=\{n \in \mathbb{N}: n>\sigma\}, \quad \mathbb{R}_{\sigma}:=[\sigma,+\infty)
$$

and the following functional spaces:

- $\mathcal{C}_{\sigma}=C([-r, \sigma] ; E)$ is the Banach space of all continuous functions

$$
\varphi:[-r, \sigma] \rightarrow E
$$

with the norm $\|\varphi\|_{\sigma}=\sup \{|\varphi(t)|: t \in[-r, \sigma]\}$.

- $\mathcal{C}_{\infty}=C([-r, \infty) ; E)$ is the Fréchet space endowed with the family of seminorms $\left\{\|\cdot\|_{n}\right\}_{n=1}^{\infty}$.

- $X_{\sigma, n}=C([\sigma, n] ; E)$ is the Banach space of all continuous functions $x:[\sigma, n] \rightarrow E$ with the norm

$$
p_{\sigma, n}(x)=\sup _{t \in[\sigma, n]}|x(t)|, \quad x \in X_{\sigma, n} .
$$

- $X_{\sigma}=C([\sigma,+\infty) ; E)$ is the Fréchet space endowed with the family of seminorms $\left\{p_{\sigma, n}\right\}_{n=1}^{\infty}$.

It is well known that $\mathcal{C}_{\infty}$ and $X_{\sigma}$ are metrizable with the metrics given respectively by

$$
\rho(x, y)=\sum_{n \in \mathbb{N}_{\sigma}}^{\infty} 2^{-n} \frac{\|x-y\|_{n}}{1+\|x-y\|_{n}}, \quad x, y \in \mathcal{C}_{\infty},
$$

and

$$
d(x, y)=\sum_{n \in \mathbb{N}_{\sigma}}^{\infty} 2^{-n} \frac{p_{\sigma, n}(x-y)}{1+p_{\sigma, n}(x-y)}, \quad x, y \in X_{\sigma} .
$$

Other notations. Let $\sigma \geq 0$ and $\varphi \in \mathcal{C}_{\sigma}$.

- For $x \in X_{\sigma, n}$ (resp. $x \in X_{\sigma}$ ), we put

$$
\begin{aligned}
& x^{\varphi}(t)= \begin{cases}x(t)+\varphi(\sigma)-x(\sigma), & t \in[\sigma, n], \\
\varphi(t), & t \in[-r, \sigma],\end{cases}
\end{aligned}
$$

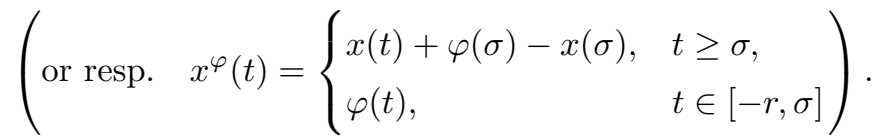

Then it is clear that $x^{\varphi} \in \mathcal{C}_{n}$ (resp. $x^{\varphi} \in \mathcal{C}_{\infty}$ ). 
- For $t \in \mathbb{R}_{+}$and $x \in X_{\sigma, n}$ (resp. $x \in X_{\sigma}$ ) we shall denote by $\xi^{\varphi}$ the map

$$
(t, x) \mapsto \xi^{\varphi}(t, x)=\left(t, x^{\varphi}(t), x_{t}^{\varphi}\right) .
$$

- Finally, if $\theta \in \mathcal{C}_{\sigma}$ (resp. $\theta \in \mathcal{C}_{\infty}$ ) then, for each $t \in[0, \sigma]$ (resp. $t \in$ $[0, \infty))$, we shall denote by $\theta_{t}$ the $\mathcal{C}_{0}$-function defined by

$$
\theta_{t}(s)=\theta(t+s), \quad s \in[-r, 0] .
$$

4.2. Some preliminaries and hypotheses. First we have the following lemmas. Their proofs are straightforward and we will omit them.

Lemma 4.1. For every $n \in \mathbb{N}_{\sigma}$ and $x, y \in X_{\sigma, n}$ we have:

(a) $\left\|x^{\varphi}\right\|_{n} \leq 2 p_{\sigma, n}(x)+\|\varphi\|_{\sigma}$,

(b) $\left|x^{\varphi}(t)-y^{\varphi}(t)\right| \leq 2 p_{\sigma, n}(x-y)$, for all $t \in[-r, n]$,

(c) $\left\|x_{t}^{\varphi}-y_{t}^{\varphi}\right\|_{0} \leq 2 p_{\sigma, n}(x-y)$, for all $t \in[0, n]$,

(d) $\left\|x_{t}^{\varphi}\right\|_{0} \leq 2 p_{\sigma, n}(x)+\|\varphi\|_{\sigma}$, for all $t \in[0, n]$.

LEMMA 4.2. The map $\xi^{\varphi}$ is continuous from $\mathbb{R}_{+} \times X_{\sigma, n}$ into $\mathbb{R}_{+} \times E \times \mathcal{C}_{n}$ (resp. from $\mathbb{R}_{+} \times X_{\sigma}$ into $\left.\mathbb{R}_{+} \times E \times \mathcal{C}_{\infty}\right)$. Moreover, we have

$$
\left\|\xi^{\varphi}(t, x)\right\| \leq t+4 p_{\sigma, n}(x)+2\|\varphi\|_{\sigma},
$$

for $(t, x) \in \mathbb{R}_{+} \times X_{\sigma, n}$ (resp. for $(t, x) \in \mathbb{R}_{+} \times X_{\sigma}$ and for all $n \in \mathbb{N}_{\sigma}$ ).

REMARK 4.3. In Lemma 4.2 as well as throughout this section the norm on a product space $M_{1} \times \ldots \times M_{k}$ is always denoted by $\|\cdot\|$ and is defined by

$$
\left\|\left(m_{1}, \ldots, m_{k}\right)\right\|=\sum_{i=1}^{k}\left\|m_{i}\right\|_{i},
$$

where $\|\cdot\|_{i}$ is the norm on $M_{i}$.

In order to study the topological structure of mild solution set for problem (1.1) we make the following assumptions:

(H1) $A$ generates an analytic semigroup $\{T(t)\}_{t \geq 0}$ such that $0 \in \rho(A)$ and $T(t)$ is compact for each $t>0$.

(H2) The function $f: \mathbb{R}_{+} \times E \times \mathcal{C}_{0} \rightarrow E$ satisfies the following conditions:

(i) the map $t \mapsto f(t, x, y)$ is measurable, for all $(x, y) \in E \times \mathcal{C}_{0}$,

(ii) the map $(x, y) \mapsto f(t, x, y)$ is continuous for almost every $t \in \mathbb{R}_{+}$,

(iii) there exists a constant $q_{1} \in[0, q)$ such that for each $C>0$, there is a nonnegative function $r_{C} \in L^{1 / q_{1}}\left(\mathbb{R}_{+}\right)$and if $(x, y) \in E \times \mathcal{C}_{0}$ with $\|(x, y)\| \leq C$ then $|f(t, x, y)| \leq r_{C}(t)$ for almost every $t \in \mathbb{R}_{+}$,

(iv) for any bounded subset $J$ of $\mathbb{R}_{+}$we have

$$
\lim _{\|(x, y)\| \rightarrow \infty} \frac{|f(t, x, y)|}{\|(x, y)\|}=0,
$$

uniformly in $t \in J$. 
(H3) $h: \mathbb{R}_{+} \times E \times \mathcal{C}_{0} \rightarrow E$ is continuous. Further, there exists a constant $\beta \in(0,1)$ and a sequence $\left\{H_{n}>0: n \in \mathbb{N}\right\}$ with

$$
H_{n}\left((M+1)\left\|A^{-\beta}\right\|_{\mathcal{L}(E)}+\frac{n^{\beta q} \Gamma(1+\beta) C_{1-\beta}}{\beta \Gamma(1+\beta q)}\right)<\frac{1}{4},
$$

such that $h \in D\left(A^{\beta}\right)$ and, for any $(x, y),\left(x^{\prime}, y^{\prime}\right) \in E \times \mathcal{C}_{0}$, the function $A^{\beta} h(\cdot, x, y)$ is measurable and $A^{\beta} h(t, \cdot, \cdot)$ satisfies the Lipschitz condition

$$
\left|A^{\beta} h(t, x, y)-A^{\beta} h\left(t, x^{\prime}, y^{\prime}\right)\right| \leq H_{n}\left(\left|x-x^{\prime}\right|+\left\|y-y^{\prime}\right\|_{0}\right),
$$

for almost every $t \in[0, n]$.

\subsection{Main result.}

ThEOREM 4.4. Let (H1)-(H3) hold. Then the mild solution set $\mathcal{S}$ of problem (1.1) is an $R_{\delta}$-set.

In order to prove Theorem 4.4 we first consider the operators

$$
\widehat{\mathcal{U}}_{n}, \widehat{\mathcal{B}}_{n}: X_{0, n} \rightarrow X_{0, n}
$$

defined, for all $t \in[0, n]$, by

$$
\begin{aligned}
& \widehat{\mathcal{U}}_{n} y(t)=S_{q}(t)\left[\psi(0)-h\left(\xi^{\psi}(0, y)\right)\right]+h\left(\xi^{\psi}(t, y)\right) \\
&+\int_{0}^{t}(t-s)^{q-1} A T_{q}(t-s) h\left(\xi^{\psi}(s, y)\right) d s, \\
& \widehat{\mathcal{B}}_{n} y(t)=\int_{0}^{t}(t-s)^{q-1} T_{q}(t-s) f\left(\xi^{\psi}(s, y)\right) d s .
\end{aligned}
$$

Lemma 4.5. For each $n \in \mathbb{N}$ we have

$$
p_{0, n}\left(\widehat{\mathcal{U}}_{n}(x)-\widehat{\mathcal{U}}_{n}(y)\right) \leq k_{n} p_{0, n}(x-y),
$$

for all $x, y \in X_{0, n}$, where

$$
k_{n}=4 H_{n}\left((M+1)\left\|A^{-\beta}\right\|_{\mathcal{L}(E)}+\frac{n^{\beta q} \Gamma(1+\beta) C_{1-\beta}}{\beta \Gamma(1+\beta q)}\right) .
$$

Proof. Let $x, y \in X_{0, n}$. For every $t \in[0, n]$, we have

$$
\begin{aligned}
\mid \widehat{\mathcal{U}}_{n} x(t) & -\widehat{\mathcal{U}}_{n} y(t) \mid \\
\leq & \left|S_{q}(t)\left[h\left(\xi^{\psi}(0, x)\right)-h\left(\xi^{\psi}(0, y)\right)\right]\right|+\left|\left[h\left(\xi^{\psi}(t, x)\right)-h\left(\xi^{\psi}(t, y)\right)\right]\right| \\
& +\left|\int_{0}^{t}(t-s)^{q-1} A T_{q}(t-s)\left[h\left(\xi^{\psi}(s, x)\right)-h\left(\xi^{\psi}(s, y)\right)\right] d s\right| .
\end{aligned}
$$

Hence, by applying Lemma 2.2, assumption (H3) and Lemma 4.1 we get

$$
\begin{aligned}
& \left|\widehat{\mathcal{U}}_{n} x(t)-\widehat{\mathcal{U}}_{n} y(t)\right| \\
& \quad \leq M H_{n}\left\|A^{-\beta}\right\|_{\mathcal{L}(E)}\left(\left|x^{\psi}(0)-y^{\psi}(0)\right|+\left\|x_{0}^{\psi}-y_{0}^{\psi}\right\|_{0}\right)
\end{aligned}
$$




$$
\begin{aligned}
& +H_{n}\left\|A^{-\beta}\right\|_{\mathcal{L}(E)}\left(\left|x^{\psi}(t)-y^{\psi}(t)\right|+\left\|x_{t}^{\psi}-y_{t}^{\psi}\right\|_{0}\right) \\
& +\left|\int_{0}^{t}(t-s)^{q-1} A^{1-\beta} T_{q}(t-s) t\left[A^{\beta} h\left(\xi^{\psi}(s, x)\right)-A^{\beta} h\left(\xi^{\psi}(s, y)\right)\right] d s\right| \\
\leq & 4 H_{n}(M+1)\left\|A^{-\beta}\right\|_{\mathcal{L}(E)} p_{0, n}(x-y) \\
& +\left[4 H_{n} \frac{q C_{1-\beta} \Gamma(1+\beta)}{\Gamma(1+q \beta)} \int_{0}^{t}(t-s)^{q \beta-1} d s\right] p_{0, n}(x-y) \\
\leq & k_{n} p_{0, n}(x-y) .
\end{aligned}
$$

This implies that $p_{0, n}\left(\widehat{\mathcal{U}}_{n}(x)-\widehat{\mathcal{U}}_{n}(y)\right) \leq k_{n} p_{0, n}(x-y)$.

LEMMA 4.6. The operator $\widehat{\mathcal{B}}_{n}$ is completely continuous and satisfies the relation

$$
\lim _{p_{0, n}(x) \rightarrow \infty} \frac{p_{0, n}\left(\widehat{\mathcal{B}}_{n}(x)\right)}{p_{0 n}(x)}=0 .
$$

Proof. The proof of this lemma consists of several steps.

Step 1. $\widehat{\mathcal{B}}_{n}$ is continuous.

Indeed, assume that $\left(x_{k}\right)$ is a sequence in $X_{0, n}$ converging to $x \in X_{0, n}$. Put $G=\left\{x_{k}: k \in \mathbb{N}\right\} \cup\{x\}$. Since $\xi^{\psi}([0, n] \times G)$ is compact there is a nonnegative function $r_{G} \in L^{1 / q_{1}}\left(\mathbb{R}_{+}\right)$such that $\left|f\left(\xi^{\psi}(s, y)\right)\right| \leq r_{G}(s)$ for all $y \in G$ and for almost every $s \in[0, n]$ by using (H2) (iii). This implies

$$
\left|f\left(\xi^{\psi}\left(s, x_{k}\right)\right)-f\left(\xi^{\psi}(s, x)\right)\right| \leq 2 r_{G}(s),
$$

for all $k \in \mathbb{N}$ and for almost every $s \in[0, n]$. On the other hand, it follows from the continuity of $\xi^{\psi}$ and assumption (H2) (ii) that $f\left(\xi^{\psi}\left(s, x_{k}\right)\right)-f\left(\xi^{\psi}(s, x)\right)$ converges to 0 , for almost every $s \in[0, n]$. Hence, by the Lebesgue dominated convergence theorem,

$$
\lim _{k \rightarrow \infty} \int_{0}^{n}\left|f\left(\xi^{\psi}\left(s, x_{k}\right)\right)-f\left(\xi^{\psi}(s, x)\right)\right|^{1 / q_{1}} d s=0 .
$$

From Lemma 2.2 and Hölder's inequality we get

$$
\begin{aligned}
\mid \widehat{\mathcal{B}}_{n} x_{k}(t) & -\widehat{\mathcal{B}}_{n} x(t) \mid \\
= & \left|\int_{0}^{t}(t-s)^{q-1} T_{q}(t-s)\left[f\left(\xi^{\psi}\left(s, x_{k}\right)\right)-f\left(\xi^{\psi}(s, x)\right)\right] d s\right| \\
\leq & \frac{q M}{\Gamma(1+q)} \int_{0}^{t}(t-s)^{q-1}\left[f\left(\xi^{\psi}\left(s, x_{k}\right)\right)-f\left(\xi^{\psi}(s, x)\right)\right] d s \\
\leq & \frac{q M}{\Gamma(1+q)}\left(\int_{0}^{t}(t-s)^{(q-1) /\left(1-q_{1}\right)} d s\right)^{1-q_{1}} \\
& \times\left(\int_{0}^{t}\left|f\left(\xi^{\psi}\left(s, x_{k}\right)\right)-f\left(\xi^{\psi}(s, x)\right)\right|^{1 / q_{1}}(s) d s\right)^{q_{1}} \\
\leq & \frac{q M n^{q-q_{1}}}{\Gamma(1+q)}\left(\frac{1-q_{1}}{q-q_{1}}\right)^{1-q_{1}}\left\|f\left(\xi^{\psi}\left(\cdot, x_{k}\right)\right)-f\left(\xi^{\psi}(\cdot, x)\right)\right\|_{L^{1 / q_{1}(0, n)}},
\end{aligned}
$$


for all $t \in[0, n]$. Here we used the following estimate:

$$
\left(\int_{0}^{t}(t-s)^{(q-1) /\left(1-q_{1}\right)} d s\right)^{1-q_{1}}=t^{q-q_{1}}\left(\frac{1-q_{1}}{q-q_{1}}\right)^{1-q_{1}} \leq n^{q-q_{1}}\left(\frac{1-q_{1}}{q-q_{1}}\right)^{1-q_{1}} .
$$

Hence $p_{0, n}\left(\widehat{\mathcal{B}}_{n} x_{k}-\widehat{\mathcal{B}}_{n} x\right)$ converges to 0 when $k \rightarrow \infty$. Therefore, $\widehat{\mathcal{B}}_{n}$ is continuous.

Step 2. $\widehat{\mathcal{B}}_{n}$ is compact.

Let $Q$ be a bounded subset of $X_{0 n}$. By using Lemma 4.2 , the set $\xi^{\psi}([0, n] \times Q)$ is bounded. Hence there is a nonnegative function $r_{Q} \in L^{1 / q_{1}}\left(\mathbb{R}_{+}\right)$such that

$$
\left|f\left(\xi^{\psi}(t, x)\right)\right| \leq r_{Q}(t),
$$

for all $x \in Q$ and for almost every $t \in[0, n]$, by using again (H2) (iii).

For $x \in Q$ and $0 \leq t_{1}<t_{2} \leq n$, we have

$$
\begin{aligned}
\left|\widehat{\mathcal{B}}_{n} x\left(t_{2}\right)-\widehat{\mathcal{B}}_{n} x\left(t_{1}\right)\right| & =\mid \int_{0}^{t_{2}}\left(t_{2}-s\right)^{q-1} T_{q}\left(t_{2}-s\right) f\left(\xi^{\psi}(s, x)\right) d s \\
& -\int_{0}^{t_{1}}\left(t_{1}-s\right)^{q-1} T_{q}\left(t_{1}-s\right) f\left(\xi^{\psi}(s, x)\right) d s \mid \leq I_{1}+I_{2}+I_{3},
\end{aligned}
$$

where

$$
\begin{aligned}
& I_{1}=\left|\int_{t_{1}}^{t_{2}}\left(t_{2}-s\right)^{q-1} T_{q}\left(t_{2}-s\right) f\left(\xi^{\psi}(s, x)\right) d s\right|, \\
& I_{2}=\left|\int_{0}^{t_{1}}\left[\left(t_{2}-s\right)^{q-1}-\left(t_{1}-s\right)^{q-1}\right] T_{q}\left(t_{2}-s\right) f\left(\xi^{\psi}(s, x)\right) d s\right|, \\
& I_{3}=\left|\int_{0}^{t_{1}}\left(t_{1}-s\right)^{q-1}\left[T_{q}\left(t_{2}-s\right)-T_{q}\left(t_{1}-s\right)\right] f\left(\xi^{\psi}(s, x)\right) d s\right| .
\end{aligned}
$$

Estimate $I_{1}$. By using Lemma 2.2 and Hölder's inequality, we have

$$
I_{1} \leq \frac{q M}{\Gamma(1+q)}\left(\frac{1-q_{1}}{q-q_{1}}\right)^{1-q_{1}}\left\|r_{Q}\right\|_{L^{1 / q_{1}(0, n)}}\left(t_{2}-t_{1}\right)^{q-q_{1}}
$$

Estimate $I_{2}$. We shall use the following inequality:

$$
\left(a^{\alpha}-b^{\alpha}\right)^{\gamma} \leq a^{\alpha \gamma}-b^{\alpha \gamma}, \text { for all } 0<a<b, \alpha<0, \gamma>1 .
$$

In order to prove this inequality we note that the the function $j(t)=\left(t^{\alpha}-1\right)^{\gamma}-$ $t^{\alpha \gamma}+1$ is increasing on $(0,1]$ by

$$
j^{\prime}(t)=\alpha \gamma\left[\left(t^{\alpha}-1\right)^{\gamma-1} t^{\alpha-1}-t^{\alpha \gamma-1}\right] \geq 0, \quad \text { for all } t \in(0,1] .
$$

Hence $j(t) \leq j(1)=0$ which implies that $\left(t^{\alpha}-1\right)^{\gamma} \leq t^{\alpha \gamma}-1$. Let $t=a / b$ then we obtain the desired inequality.

By using the above inequality, Lemma 2.2 and Hölder's inequality, we get

$$
I_{2} \leq \frac{q M}{\Gamma(1+q)}\left\|r_{Q}\right\|_{L^{1 / q_{1}(0, n)}}\left(\int_{0}^{t_{1}}\left[\left(t_{2}-s\right)^{q-1}-\left(t_{1}-s\right)^{q-1}\right]^{1 /\left(1-q_{1}\right)} d s\right)^{1-q_{1}}
$$




$$
\begin{aligned}
\leq & \frac{q M}{\Gamma(1+q)}\left(\frac{1-q_{1}}{q-q_{1}}\right)^{1-q_{1}}\left\|r_{Q}\right\|_{L^{1 / q_{1}}(0, n)}\left(t_{1}^{\left(q-q_{1}\right) /\left(1-q_{1}\right)}\right. \\
& \left.-t_{2}^{\left(q-q_{1}\right) /\left(1-q_{1}\right)}+\left(t_{2}-t_{1}\right)^{\left(q-q_{1}\right) /\left(1-q_{1}\right)}\right)^{1-q_{1}} \\
\leq & \frac{q M}{\Gamma(1+q)}\left(\frac{1-q_{1}}{q-q_{1}}\right)^{1-q_{1}}\left\|r_{Q}\right\|_{L^{1 / q_{1}(0, n)}}\left(t_{2}-t_{1}\right)^{q-q_{1}} .
\end{aligned}
$$

Estimate $I_{3}$. Without loss of generality we assume that $t_{1}>0$. For $\varepsilon>0$ small enough, we have

$$
\begin{aligned}
I_{3} \leq & \int_{0}^{t_{1}-\varepsilon}\left(t_{1}-s\right)^{q-1}\left|T_{q}\left(t_{2}-s\right) f\left(\xi^{\psi}(s, x)\right)-T_{q}\left(t_{1}-s\right) f\left(\xi^{\psi}(s, x)\right)\right| d s \\
& +\int_{t_{1}-\varepsilon}^{t_{1}}\left(t_{1}-s\right)^{q-1}\left|T_{q}\left(t_{2}-s\right) f\left(\xi^{\psi}(s, x)\right)-T_{q}\left(t_{1}-s\right) f\left(\xi^{\psi}(s, x)\right)\right| d s \\
\leq & \left(\frac{1-q_{1}}{q-q_{1}}\right)^{1-q_{1}}\left\|r_{Q}\right\|_{L^{1 / q_{1}[0, n]}}\left[\frac{2 q M \varepsilon^{1-q_{1}}}{\Gamma(1+q)}\right. \\
& \left.+\left(t_{1}^{\left(q-q_{1}\right) /\left(1-q_{1}\right)}-\varepsilon^{\left(q-q_{1}\right) /\left(1-q_{1}\right)}\right)^{1-q_{1}} \sup _{s \in\left[0, t_{1}-\varepsilon\right]}\left\|T_{q}\left(t_{2}-s\right)-T_{q}\left(t_{1}-s\right)\right\|_{\mathcal{L}(E)}\right] .
\end{aligned}
$$

Since $T(t)$ is compact, and from Lemma 2.2, it follows that $T_{q}(t)(t>0)$ is continuous in $t$ in the uniform operator topology. Hence $I_{3}$ converges to zero independently of $x \in Q$ as $t_{2}-t_{1} \rightarrow 0$ and $\varepsilon \rightarrow 0$.

Combining the above estimates, we can conclude that $\left\{\widehat{\mathcal{B}}_{n} x: x \in Q\right\}$ is equicontinuous.

For each $t \in[0, n]$ we put $\mathcal{K}(t)=\left\{\widehat{\mathcal{B}}_{n} x(t): x \in Q\right\}$. It is sufficient to prove the relative compactness in $E$ of $\mathcal{K}(t)$. For this, for $\varepsilon \in(0, t)$ and $\delta>0$, we define an operator $\widehat{\mathcal{B}}_{n}^{\varepsilon}$ on $Q$ by

$$
\widehat{\mathcal{B}}_{n}^{\varepsilon} x(t)=\int_{0}^{t-\varepsilon} \int_{\delta}^{\infty} q \theta(t-s)^{q-1} \phi_{q}(\theta) T\left[(t-s)^{q} \theta\right] f\left(\xi^{\psi}(s, x)\right) d \theta d s,
$$

for $x \in Q$ and $t \in[0, n]$. It is not difficult to see that

$$
\widehat{\mathcal{B}}_{n}^{\varepsilon} x(t)=T\left(\varepsilon^{q} \delta\right) q \int_{0}^{t-\varepsilon} \int_{\delta}^{\infty} \theta(t-s)^{q-1} \phi_{q}(\theta) T\left[(t-s)^{q} \theta-\varepsilon^{q} \delta\right] f\left(\xi^{\psi}(s, x)\right) d \theta d s .
$$

By using the assumptions on $f$ and the boundedness of $Q$, it follows that the set

$$
\left\{q \int_{0}^{t-\varepsilon} \int_{\delta}^{\infty} \theta(t-s)^{q-1} \phi_{q}(\theta) T\left[(t-s)^{q} \theta-\varepsilon^{q} \delta\right] f\left(\xi^{\psi}(s, x)\right) d \theta d s: x \in Q\right\}
$$

is bounded in $E$. Then, from the compactness of $T\left(\varepsilon^{q} \delta\right)$, we can claim that $\mathcal{K}^{\varepsilon}(t)=\left\{\widehat{\mathcal{B}}_{n}^{\varepsilon} x(t): x \in Q\right\}$ is relatively compact in $E$. On the other hand, since

$$
T_{q}(t) y=q \int_{0}^{\infty} \theta \phi(\theta) T\left(t^{q} \theta\right) y d \theta, \quad y \in E,
$$

we deduce that, for every $x \in Q$,

$$
\left|\widehat{\mathcal{B}}_{n} x(t)-\widehat{\mathcal{B}}_{n}^{\varepsilon} x(t)\right|=q \mid \int_{0}^{t} \int_{0}^{\delta} \theta(t-s)^{q-1} \phi_{q}(\theta) T\left((t-s)^{q} \theta\right) f\left(\xi^{\psi}(s, x)\right) d \theta d s
$$




$$
\begin{aligned}
& \quad+\int_{0}^{t} \int_{\delta}^{\infty} \theta(t-s)^{q-1} \phi_{q}(\theta) T\left((t-s)^{q} \theta\right) f\left(\xi^{\psi}(s, x)\right) d \theta d s \\
& \quad-\int_{0}^{t-\varepsilon} \int_{\delta}^{\infty} \theta(t-s)^{q-1} \phi_{q}(\theta) T\left[(t-s)^{q} \theta\right] f\left(\xi^{\psi}(s, x)\right) d \theta d s \mid \\
& \leq q\left|\int_{0}^{t} \int_{0}^{\delta} \theta(t-s)^{q-1} \phi_{q}(\theta) T\left((t-s)^{q} \theta\right) f\left(\xi^{\psi}(s, x)\right) d \theta d s\right| \\
& \quad+q\left|\int_{t-\varepsilon}^{t} \int_{\delta}^{\infty} \theta(t-s)^{q-1} \phi_{q}(\theta) T\left[(t-s)^{q} \theta\right] f\left(\xi^{\psi}(s, x)\right) d \theta d s\right| \\
& \leq q\left(J_{1}+J_{2}\right) .
\end{aligned}
$$

By using Hölder's inequality, we estimate $J_{1}$ and $J_{2}$ as follows:

$$
\begin{aligned}
J_{1} & \leq M\left(\int_{0}^{t}(t-s)^{q-1} r_{Q}(s) d s\right)\left(\int_{0}^{\delta} \theta \phi_{q}(\theta) d \theta\right) \\
& \leq M\left(\int_{0}^{t}(t-s)^{(q-1) /\left(1-q_{1}\right)} d s\right)^{1-q_{1}}\left(\int_{0}^{t} r_{Q}^{1 / q_{1}}(s) d s\right)^{q_{1}}\left(\int_{0}^{\delta} \theta \phi_{q}(\theta) d \theta\right) \\
& \leq M\left(\frac{1-q_{1}}{q-q_{1}}\right)^{1-q_{1}} n^{q-q_{1}}\left\|r_{Q}\right\|_{L^{1 / q_{1}}[0, n]}\left(\int_{0}^{\delta} \theta \phi_{q}(\theta) d \theta\right),
\end{aligned}
$$

and

$$
\begin{aligned}
J_{2} & \leq M\left(\int_{t-\varepsilon}^{t}(t-s)^{q-1} r_{Q}(s) d s\right)\left(\int_{\delta}^{\infty} \theta \phi_{q}(\theta) d \theta\right) \\
& \leq M\left(\int_{t-\varepsilon}^{t}(t-s)^{(q-1) /\left(1-q_{1}\right)} d s\right)^{1-q_{1}}\left(\int_{t-\varepsilon}^{t} r_{Q}^{1 / q_{1}}(s) d s\right)^{q_{1}}\left(\int_{\delta}^{\infty} \theta \phi_{q}(\theta) d \theta\right) \\
& \leq M\left(\frac{1-q_{1}}{q-q_{1}}\right)^{1-q_{1}} \varepsilon^{q-q_{1}}\left\|r_{Q}\right\|_{L^{1 / q_{1}}[0, n]}\left(\int_{0}^{\infty} \theta \phi_{q}(\theta) d \theta\right) .
\end{aligned}
$$

Combining above inequalities and noting that

$$
\int_{0}^{\infty} \theta \phi_{q}(\theta) d \theta=\frac{1}{\Gamma(q+1)}
$$

(see [28]), we obtain

$$
\begin{aligned}
\mid \widehat{\mathcal{B}}_{n} x(t) & -\widehat{\mathcal{B}}_{n}^{\varepsilon} x(t) \mid \\
& \leq q M\left(\frac{1-q_{1}}{q-q_{1}}\right)^{1-q_{1}}\left\|r_{Q}\right\|_{L^{1 / q_{1}}[0, n]}\left[n^{q-q_{1}} \int_{0}^{\delta} \theta \phi_{q}(\theta) d \theta+\frac{\varepsilon^{q-q_{1}}}{\Gamma(1+q)}\right] .
\end{aligned}
$$

Hence there are relatively compact sets arbitrarily close to the set $\mathcal{K}(t)$. So $\mathcal{K}(t)$ is also relatively compact in $E$.

Step 3. Let $\varepsilon>0$. It follows from (H2) (iv) that there exists a positive constant $C$ such that

$$
|f(t, x, y)| \leq \frac{\Gamma(1+q) \varepsilon}{4 M n^{q}}\|(x, y)\|
$$


for all $t \in[0, n]$ and for all $(x, y) \in E \times \mathcal{C}_{0}$ with $\|(x, y)\|>C$. Let $r_{C} \in L^{1 / q_{1}}\left(\mathbb{R}_{+}\right)$ be a nonnegative function satisfying

$$
|f(t, x, y)| \leq r_{C}(t), \quad \text { for a.e. } t \in \mathbb{R}_{+},
$$

provided that $\|(x, y)\| \leq C$. Hence, for all $(x, y) \in E \times \mathcal{C}_{0}$,

$$
|f(t, x, y)| \leq r_{C}(t)+\frac{\Gamma(1+q) \varepsilon}{4 M n^{q}}\|(x, y)\|, \quad \text { a.e. } t \in[0, n] .
$$

By using Lemma 4.1, for $x \in X_{0, n}$ and $t \in[0, n]$, we have

$$
\begin{aligned}
\left|\widehat{\mathcal{B}}_{n} x(t)\right| \leq & \frac{q M}{\Gamma(1+q)} \int_{0}^{t}(t-s)^{q-1}\left|f\left(\xi^{\psi}(s, x)\right)\right| d s \\
\leq & \frac{q M}{\Gamma(1+q)} \int_{0}^{t}(t-s)^{q-1}\left[r_{C}(s)+\frac{\Gamma(1+q) \varepsilon}{4 M n^{q}}\left(\left|x^{\psi}(s)\right|+\left\|x_{s}^{\psi}\right\|_{n}\right)\right] d s \\
\leq & \frac{q M}{\Gamma(1+q)} \int_{0}^{t}(t-s)^{q-1}\left[r_{C}(s)+\frac{\Gamma(1+q) \varepsilon}{4 M n^{q}}\left(4 p_{0, n}(x)+2\|\psi\|_{0}\right)\right] d s \\
\leq & \frac{q M}{\Gamma(1+q)}\left[\left(\frac{1-q_{1}}{q-q_{1}}\right)^{1-q_{1}} n^{q-q_{1}}\left\|r_{C}\right\|_{L^{1 / q_{1}}[0, n]}\right. \\
& \left.+\frac{\Gamma(1+q) \varepsilon}{4 q M}\left(4 p_{0, n}(x)+2\|\psi\|_{0}\right)\right] \\
\leq & \varepsilon p_{0, n}(x)+\frac{q M}{\Gamma(1+q)}\left(\frac{1-q_{1}}{q-q_{1}}\right)^{1-q_{1}} n^{q-q_{1}}\left\|r_{C}\right\|_{L^{1 / q_{1}}[0, n]}+\frac{\varepsilon}{2}\|\psi\|_{0} .
\end{aligned}
$$

So $\lim _{p_{0, n}(x) \rightarrow \infty} p_{0, n}\left(\widehat{\mathcal{B}}_{n}(x)\right) / p_{0, n}(x) \leq \varepsilon$. This shows that

$$
\lim _{p_{0, n}(x) \rightarrow \infty} \frac{p_{0, n}\left(\widehat{\mathcal{B}}_{n}(x)\right)}{p_{0, n}(x)}=0
$$

because $\varepsilon$ is arbitrary.

Lemma 4.7. Let (H1)-(H3) hold. Then, for every $n \in \mathbb{N}$, the set of fixed points of operator $\widehat{\mathcal{U}}_{n}+\widehat{\mathcal{B}}_{n}$, denoted by $\operatorname{Fix}\left(\widehat{\mathcal{U}}_{n}+\widehat{\mathcal{B}}_{n}\right)$, is $R_{\delta}$.

Proof. It is clear that $\widehat{\mathcal{B}_{n}} x(0)=0$ for all $x \in X_{0, n}$. Let $\varepsilon \in(0, n]$. Assume that $x, y \in X_{0, n}$ and satisfy

$$
\left.x\right|_{I_{\varepsilon}}=\left.y\right|_{I_{\varepsilon}}, \quad I_{\varepsilon}=[0, n] \cap[-\varepsilon, \varepsilon]=[0, \varepsilon] .
$$

By definition of $x^{\psi}$ and $y^{\psi}$, we have $x^{\psi}(t)=y^{\psi}(t)$ and $x_{t}^{\psi}=y_{t}^{\psi}$ for all $t \in I_{\varepsilon}$. This implies

$$
\left.\widehat{\mathcal{U}}_{n}(x)\right|_{I_{\varepsilon}}=\left.\widehat{\mathcal{U}}_{n}(y)\right|_{I_{\varepsilon}},\left.\quad \widehat{\mathcal{B}_{n}}(x)\right|_{I_{\varepsilon}}=\left.\widehat{\mathcal{B}_{n}}(y)\right|_{I_{\varepsilon}} .
$$

So it follows from Lemmas 4.5, 4.6 and Corollary 3.3 that $\operatorname{Fix}\left(\widehat{\mathcal{U}}_{n}+\widehat{\mathcal{B}}_{n}\right)$ is $\mathrm{R}_{\delta}$.

Next, for $\sigma \geq 0$ and $\varphi \in \mathcal{C}_{\sigma}$, we consider two operators $\mathcal{U}_{\sigma}^{\varphi}, \mathcal{B}_{\sigma}^{\varphi}: X_{\sigma} \rightarrow X_{\sigma}$ defined respectively by

$$
\mathcal{U}_{\sigma}^{\varphi} x(t)=S_{q}(t)\left[\varphi(0)-h\left(\xi^{\varphi}(0, x)\right)\right]+h\left(\xi^{\varphi}(t, x)\right)
$$




$$
\begin{aligned}
& +\int_{0}^{t}(t-s)^{q-1} A T_{q}(t-s) h\left(\xi^{\varphi}(s, x)\right) d s, \\
\mathcal{B}_{\sigma}^{\varphi} x(t) & =\int_{0}^{t}(t-s)^{q-1} T_{q}(t-s) f\left(\xi^{\varphi}(s, x)\right) d s,
\end{aligned}
$$

for all $t \geq \sigma$. We denote by

- $\Sigma_{\sigma}^{\varphi}=\operatorname{Fix}\left(\mathcal{U}_{\sigma}^{\varphi}+\mathcal{B}_{\sigma}^{\varphi}\right)$ the set of fixed points of $\mathcal{U}_{\sigma}^{\varphi}+\mathcal{B}_{\sigma}^{\varphi}$,

- $\mathcal{S}$ the mild solution set of problem (1.1).

Lemma 4.8. Suppose that $\Sigma_{0}^{\psi} \neq \emptyset$. The map $\Pi:\left(\Sigma_{0}^{\psi}, d\right) \rightarrow(\mathcal{S}, \rho)$ defined by $\Pi(x)=x^{\psi}$ is an isometry.

Proof. Let $x \in \Sigma_{0}^{\psi}$. It is easy to see that $x(0)=\psi(0)$ which implies

$$
\begin{cases}x(t) & \text { for } t \geq 0, \\ \psi(t) & \text { for } t \in[-r, 0] .\end{cases}
$$

Hence $x^{\psi} \in \mathcal{S}$. On the other hand, if $y \in \mathcal{S}$ then the restriction $x$ of $y$ on $[0, \infty)$ is an element of $\Sigma_{0}^{\psi}$ and satisfies $x^{\psi}=y$. Further, it is clear that $\Pi$ is an injection. Finally, if $x, y \in \Sigma_{0}^{\psi}$ then $\left\|x^{\psi}-y^{\psi}\right\|_{n}=p_{0, n}(x-y)$, for all $n \in \mathbb{N}$. Hence $\Pi$ is an isometry.

Finally, we shall prove Theorem 4.4.

Proof of Theorem 4.4. By Lemma 4.8, we need only to prove that $\Sigma_{0}^{\psi}$ is an $\mathrm{R}_{\delta}$-set.

Step 1. We shall prove that $\Sigma_{\sigma}^{\varphi} \neq \emptyset$ for any $\sigma \geq 0$ and $\varphi \in \mathcal{C}_{\sigma}$.

Similarly as the proofs of Lemmas 4.5 and 4.6 , we can deduce that, for each $n \in \mathbb{N}_{\sigma}$,

$$
\begin{gathered}
p_{\sigma, n}\left(\mathcal{U}_{\sigma}^{\varphi}(x)-\mathcal{U}_{\sigma}^{\varphi}(y)\right) \leq k_{n} p_{\sigma, n}(x-y), \quad \text { for all } x, y \in X_{\sigma}, \\
\lim _{p_{\sigma, n}(x) \rightarrow \infty} \frac{p_{\sigma, n}\left(\mathcal{B}_{\sigma}^{\varphi}(x)\right)}{p_{\sigma, n}(x)}=0 .
\end{gathered}
$$

Now we will check the complete continuity of the operator $\mathcal{B}_{\sigma}^{\varphi}$. Let $\left(x_{m}\right)$ be a sequence in $X_{\sigma}$ converging to $x \in X_{\sigma}$. Let $n \in \mathbb{N}_{\sigma}$ be fixed and put $G=\left\{x_{m}: m \in \mathbb{N}\right\} \cup\{x\}$. It is clear that $G$ is bounded in $X_{\sigma}$ with respect to the seminorm $p_{\sigma, n}$. By using Lemma 4.2 , we can deduce that $\xi^{\varphi}([\sigma, n] \times G)$ is also bounded in $\mathbb{R}_{+} \times E \times \mathcal{C}_{\infty}$. Here we use the seminorm $\|\cdot\|_{n}$ on $\mathcal{C}_{\infty}$. Then, similarly as in Step 1 in the proof of Lemma 4.6 we can show that

$$
\lim _{m \rightarrow \infty} p_{0 n}\left(\mathcal{B}_{\sigma}^{\varphi} x_{m}-\mathcal{B}_{\sigma}^{\varphi} x\right)=0 .
$$

Since $n$ is arbitrary it shows that $\mathcal{B}_{\sigma}^{\varphi}$ is continuous.

It remains to show that $\mathcal{B}_{\sigma}^{\varphi}$ maps bounded sets into relatively compact sets. To prove this fact we note that (see [17, Proposition 1]), for a bounded set $\Omega$ 
in $X_{\sigma}$, the set $\mathcal{B}_{\sigma}^{\varphi}(\Omega)$ is relatively compact in $X_{\sigma}$ if and only if for each $n \in \mathbb{N}$, we have

- $\left\{\left.\mathcal{B}_{\sigma}^{\varphi}(x)\right|_{[0, n]}: x \in \Omega\right\}$ is equicontinuous in $X_{0, n}$, and

- $\left\{\mathcal{B}_{\sigma}^{\varphi} x(t): x \in \Omega\right\}$ is relatively compact in $E$ for arbitrary $t \in[0, n]$.

But these claims can be proved by using similar arguments as in Step 2 in the proof of Lemma 4.6. Therefore the operator $\mathcal{B}_{\sigma}^{\varphi}$ is completely continuous. So it follows from Lemma 2.4 that

$$
\Sigma_{\sigma}^{\varphi}=\operatorname{Fix}\left(\mathcal{U}_{\sigma}^{\varphi}+\mathcal{B}_{\sigma}^{\varphi}\right) \neq \emptyset .
$$

Step 2. For each $n \in \mathbb{N}$, the set $\Lambda_{n}=\left\{\left.x\right|_{[0, n]}: x \in \Sigma_{0}^{\psi}\right\}$ is $\mathrm{R}_{\delta}$.

First we shall prove the relation $\Lambda_{n}=\operatorname{Fix}\left(\widehat{\mathcal{U}}_{n}+\widehat{\mathcal{B}}_{n}\right)$. Indeed, it is easy to check that if $y=\left.x\right|_{[0, n]} \in \Lambda_{n}$ with $x \in \Sigma_{0}^{\psi}$, then $y$ belongs to $\operatorname{Fix}\left(\widehat{\mathcal{U}}_{n}+\widehat{\mathcal{B}}_{n}\right)$. Now let $y \in \operatorname{Fix}\left(\widehat{\mathcal{U}}_{n}+\widehat{\mathcal{B}}_{n}\right)$. Then $y^{\psi} \in \mathcal{C}_{n}$. It follows from Step 1 that

$$
\Sigma_{n}^{y^{\psi}}=\operatorname{Fix}\left(\mathcal{U}_{n}^{y^{\psi}}+\mathcal{B}_{n}^{y^{\psi}}\right) \neq \emptyset .
$$

Hence there exists $x^{*} \in \Sigma_{n}^{y^{\psi}}$. Define the function $x:[0, \infty) \rightarrow E$ by

$$
x(t)= \begin{cases}y(t) & \text { for } t \in[0, n], \\ x^{*}(t) & \text { for } t \geq n .\end{cases}
$$

Then it is not difficult to check that $x \in \sigma_{0}^{\psi}$ and $\left.x\right|_{[0, n]}=y$. This shows that $y \in \Lambda_{n}$. Therefore

$$
\Lambda_{n}=\operatorname{Fix}\left(\widehat{\mathcal{U}}_{n}+\widehat{\mathcal{B}}_{n}\right) .
$$

Finally we apply Lemma 4.7 to obtain the $\mathrm{R}_{\delta}$ property of $\Lambda_{n}$.

Step 3. Since $\Sigma_{0}^{\psi}$ is inverse limit of $\Lambda_{n}$ it follows from [13, Theorem 3.9] that $\Sigma_{0}^{\psi}$ is an $\mathrm{R}_{\delta}$-set.

Acknowledgements. The authors would like to thank the anonymous referees for their useful comments and suggestions which allowed to improve this paper.

\section{REFERENCES}

[1] J. Andres And L. Górniewicz, Topology Fixed Point Priciples for Boundary Value Problems, Topol. Fixed Point Theory Appl., Kluwer, Dordrecht, 2003.

[2] P.N. Aronszajn, Le correspondant topologique de l'unicité dans la theorie des equations différentielles, Ann. Math. 43 (1942), 730-78.

[3] F.E. Browder ANd C.P. GuPtA, Topological degree and nonlinear mappings of analytic type in Banach space, J. Math. Anal. Appl. 26 (1969), 390-402.

[4] D. Baleanu and O.G. Mustafa, On the global existence of solutions to a class of fractional differential equations, Comput. Math. Appl. 59 (5) (2010), 835-1841.

[5] D. Baleanu, O.G. Mustafa and R.P. Agarwal, An existence result for a superlinear fractional differential equation, Appl. Math. Lett. 23 (9) (2010), 1129-1132. 
[6] D. Bugajewska, On the structure of solution sets of differential equations in Banach spaces, Math. Slovaca 50 (2000), 463-471.

[7] M. Cichoń And I. Kubiaczyk, Some remarks on the structure of the solution set for differential inclusions in Banach spaces, J. Math. Anal. Appl. 233 (1999), 597-606.

[8] F.S. De Blasi and J. Myjak, On the solution sets for differential inclusion, Bull. Pol. Acad. Sci. Math. 12 (1985), 17-23.

[9] S.D. Eidelman And A.N. Kochubei, Cauchy problem for fractional diffusion equations, J. Differential Equations 199 (2004), 211-255.

[10] M.M. El-Borai, Semigroups and some nonlinear fractional differential equations, Appl. Math. Comput. 149 (2004), 823-831.

[11] Some probability densities and fundamental solutions of fractional evolution equations, Chaos Solitons Fractals 149 (2004), 823-831.

[12] X. Fu AND K. EzzinBi, Existence of solutions for neutral differential evolution equations with nonlocal conditions, Nonlinear Anal. 54 (2003), 215-227.

[13] G. Gabor, On the acyclicity of fixed point sets of multivalued maps, Topol. Methods Nonlinear Anal. 14 (1999), 327-343.

[14] L. Górniewicz, Topological structure of solution sets: current results, Arch. Math. (Brno) 36 (2000), 343-382.

[15] _ Solving equations by topological methods, Opuscula Math. 25 (2005), 195-225.

[16] L.H. HoA AND K. SCHMitT, Fixed point theorems of Krasnosel'skii type in locally convex space and applications to integral equation, Results Math. 25 (1994), 291-313.

[17] _ Periodic solutions of functional-differential equations of retarded and neutral types in Banach spaces, Bound. Value Probl. Funct. Differential Equations (1995), 177185.

[18] S. Ji AND G. Li AND M. WANG, Controllability of impulsive differential systems with nonlocal conditions, Appl. Math. Comput. 217 (2011), 6981-6989.

[19] O.K. Jardat, A. Al-Omari And S. Momani, Existence of the mild solution for fractional semilinear initial value problems, Nonlinear Anal. 69 (9) (2008), 3153-3159.

[20] W. KRYsZEwski, Topological structure of solution sets of differential inclusions: the constrained case, Abstr. Appl. Anal. (2003), 325-351.

[21] Z. Kuвасек, On the structure of fixed point sets of some compact maps in the Fréchet space, Math. Bohem. 118 (1993), 343-358.

[22] J.J. Nieto, Aronszajn's theorem for some nonlinear Dirichlet problems with unbounded nonlinearities, Proc. Edinburgh Math. Soc. 31 (1988), 345-351.

[23] J.P.C. Santos, M.M. Aruunan and C. Cuevas, Existence results for fractional neutral integro-differential equations with state dependent delay, Comput. Math. Appl. 62 (2011), $1275-1283$.

[24] J.P.C. Santos, V. Vijayakumar and R. Murugesu, Existence of mild solutions for nonlocal Cauchy problem for fractional neutral integro-differential equation with unbounded delay, Commun. Math. Anal. 14 (2013), 59-71.

[25] A. PAZY, Semigroups of Linear Operators and Applications to Partial Differential Equations, Springer, New York, 1983.

[26] J.R. WAnG, Z. FAn and Y. Zhou, Nonlocal controllability of semilinear dynamic systems with fractional derivative in Banach spaces, J. Optim. Theory Appl. 154 (2012), 292-302.

[27] X. Zheng And X. YAng, The structure of weak Pareto solution sets in piecewise linear multiobjective optimization in normed spaces, E. Sci. China Ser. A 51 (2008), 1243-1256. 
[28] Y. Zhou AND F. JiAO, Existence of mild solutions for fractional neutral evolution equations, Comput. Math. Appl. 59 (2010), 1063-1077.

Manuscript received March 3, 2015

accepted October 4,2015

Le Hoan Hoa and Nguyen Ngoc Trong

Department of Mathematics

University of Pedagogy HoChiMinh City

280 An Duong Vuong Str, District 5

HoChiMinh city, VIETNAM

E-mail address: trongnn37@gmail.com

Le Xuan Truong

Department of Mathematics and Statistics University of Economics HoChiMinh City

59C, Nguyen Dinh Chieu Str, District 3

HoChiMinh city, VIETNAM

E-mail address: lxuantruong@gmail.com 